\title{
Editorial
}

\section{Guest editor's forewords: Special issue on Valuetools 2017}

Valuetools, the EAI International Conference on Performance Evaluation Methodologies and Tools, has grown in recent years as a popular forum focusing on the latest developments in performance evaluation techniques and related tool support. This virtual special issue consists of 6 papers extending earlier versions presented at Valuetools 2017, the 11th edition of the conference, which was held in Venice on December 5-7, 2017. A selection of the top-ranked conference papers was chosen by the chairs and the authors were invited to submit an extended version to this special issue. The journal review process included both members of the Valuetools program committee and additional reviewers that were not involved in the conference refereeing process. The resulting collection of papers comprises exciting developments in performance evaluation that we hope will engage the reader.

In the paper Markov chains with perturbed rates to absorption: Theory and application to model repair [1], Gouberman, Siegle and Tati study the effects of perturbation in the transition rates of absorbing Markov chains. They analyze in particular the question of monotonicity of hitting probabilities. While this does not hold in general, the authors find that the certain enveloping functions defined as the maximum or minimum of a given set of hitting probabilities are instead monotonic. The applicability of their results spans among others repairmen systems, reliability analysis, and stochastic logic.

In Capacity expansion of neutral ISPs via content provider participation: The bargaining edge [2], Kalvit and coauthors models incentives for infrastructure investment by Infrastructure Service Providers (ISPs). Three voluntary contribution models for content providers are considered, where they can contribute to the ISP infrastructure upgrade costs. The authors conclude that a bargaining model produces a greater incentive for the ISP to invest in infrastructure. Some interesting recommendations for policy makers are drawn out of this analysis.

In FCFS parallel service systems and matching models [3], Adan, Kleiner, Righter and Weiss analyze three parallel service models in which customers of several types are served by several types of servers subject to a bipartite compatibility graph. The authors study the relationships between these three models, and show that they are closely related to the first come first served (FCFS) infinite bipartite matching model. A generalization of Burke's theorem to parallel service systems is presented by introducing a directed bipartite matching model in which the queueing systems are embedded.

In Scalable analytical model for reliability measures in aging VLSI by interacting Markovian agents [4], Cerotti, Miele, Gribaudo, Bobbio and Bolchini present an analytical framework based on an Markovian Agent Model (MAM) for aging effects in VLSI systems. The paper presents the MAM formalism and discusses how a fairly general aging model can be built with this formalism. The flexibility and the effectiveness of the model are illustrated by computing performance-reliability related measures on two case studies: a Multi-Core System-on-Chip and a Solid State Drive.

In Stochastic approximation algorithms for rumor source inference on graphs [5], Kalvit, Borkar and Karamchandani address the problem of detecting the source of rumor in a network. The main contribution is the development of a class of estimators which are agnostic to the stochastic model which underlies the rumor spreading and generalises previous works on the subject. The simulations performed on different types of graphs show encouraging outcomes for what concerns the robustness and performance of the proposed approach.

Finally, in DeepComNet: Performance evaluation of network topologies using graph-based deep learning [6], Geyer proposes a new machine-learning based approach to predict network performance. The method resorts to Graph Gated Neural Networks and, according to the case study, provides accurate performance predictions based only on a graph-based representation of network topologies.

\section{References}

[1] Alexander Gouberman, Markus Siegle, Bharath Tati, Markov chains with perturbed rates to absorption: Theory and application to model repair, Perform. Eval. 130 (2019) 32-50. 
[2] Anand Kalvit, Saurabh Pinjani, Gaurav Kasbekar, D. Manjunath, Jayakrishnan Nair, Capacity expansion of neutral ISPs via content provider participation: The bargaining edge, Perform. Eval. 133 (2019) 43-56.

[3] Ivo Adan, Igor Kleiner, Rhonda Righter, Gideon Weiss, FCFS parallel service systems and matching models, Perform. Eval. 127-128 (2018) $253-272$.

[4] Davide Cerotti, Antonio Miele, Marco Gribaudo, Andrea Bobbio, Cristiana Bolchini, Scalable analytical model for reliability measures in aging VLSI by interacting Markovian agents, Perform. Eval. 132 (2019) 21-37.

[5] Anand Kalvit, Vivek S. Borkar, Nikhil Karamchandani, Stochastic approximation algorithms for rumor source inference on graphs, Perform. Eval. $132(2019) 1-20$.

[6] Fabien Geyer, DeepComNet: Performance evaluation of network topologies using graph-based deep learning, Perform. Eval. 130 (2019) 1-16.

Andrea Marin

Giuliano Casale

Dorina C. Petriu

Sabina Rossi 